\title{
Determination of inertial parameters using a dynamometer
}

\author{
Jongsang Son, Jeseong Ryu, Jungyoon Kim and Youngho Kim \\ Department of Biomedical Engineering and Institute for Convergence Study of Bio-Medical Wellness, \\ Yonsei University (Wonju Campus), Maeji-ri, Heungeop-myeon, Wonju-si, Gangwon-do 220-710, \\ Republic of Korea
}

\begin{abstract}
In this study, a simple method based on the dynamic equation of motion was introduced to determine the moment of inertia using a commercial dynamometer, and an optimization technique was utilized to estimate inertial parameters with the determined moment of inertia. To evaluate the feasibility of the developed method, three different passive speeds (i.e. 240,270 and $300 \%$ s) were chosen to confirm whether the moment of inertia values are the same irrespective of angular speeds. Moreover, the estimated inertial parameters (i.e., the mass, center of mass and moment of inertia) of the elbow attachment and the disk-like $3 \mathrm{~kg}$-weight were compared with solutions of uniform square cube and solid disk, respectively. As a result, the values of moments of inertia of the elbow attachment were $0.216 \pm 0.017,0.215 \pm 0.016$ and $0.216 \pm 0.017$ $\mathrm{kg} \cdot \mathrm{m}^{2}$ at angular speeds of 240,270 and $300^{\circ} \mathrm{s}$, respectively. The values of the moment of inertia of both the attachment and weight were $0.821 \pm 0.054,0.823 \pm 0.058$ and $0.824 \pm 0.053 \mathrm{~kg} \cdot \mathrm{m}^{2}$ at angular speeds of 240,270 and $300 \%$ s, respectively. There were no significant differences among the speeds. The estimated inertial parameters of the attachment or the weight were very similar to the theoretical values. Therefore, it is expected that the developed method has the potential to estimate inertial parameters of a human body segment and to improve the accuracy and reliability of the studies on human dynamics.
\end{abstract}

Keywords: Inertial parameters, mass, center of mass, radius of gyration, moment of inertia

\section{Introduction}

Link-segment models are widely used to analyze joint kinematic and kinetic variables (i.e., joint trajectories, forces, moments, or powers). Such model represents the human body as mechanically interconnected rigid body segments, whose inertial parameters such as the mass, center of mass and moment of inertia are essential for analyzing human movements. Since each inertial parameter is obviously a property of a segment, those parameters are required to be customized according to individual subjects. Nonetheless, values of inertial parameters of the body segment have little consistency among different studies, and can even vary by more than 40\% [1]. Andrews and Mish [2] suggested that a small percent of variations in the parameters could propagate into considerably large variations (approximately $7 \%$ in internal forces and $12 \%$ in joint moments) through a sensitivity analysis of two-

\footnotetext{
*Corresponding author: Youngho Kim, Department of Biomedical Engineering and Institute for Convergence Study of Bio-Medical Wellness, Yonsei University (Wonju Campus), Maeji-ri, Heungeop-myeon, Wonju-si, Gangwon-do 220-710, Republic of Korea. Tel.: +82-33-760-2492; Fax: +82-33-760-2852; E-mail: younghokim@yonsei.ac.kr.
}

0959-2989/14/\$27.50 @ 2014 - IOS Press and the authors. 
dimensional (sagittal plane) inverse dynamics with the parameters varying within $\pm 5 \%$. Thus, an accurate estimation of inertial parameters is needed to analyze human movements better [3].

In order to determine inertial parameters, many approaches have been introduced. Linear [4-8], nonlinear [9-11] or multiple [12-16] regression equations were obtained from the direct measurement of cadavers $[4,17,18]$ or living subjects $[5-16,19]$ using various measurement modalities such as simple reaction boards $[4,19]$, magnetic resonance imaging [18,20], computerized tomography [17] and three-dimensional (3D) laser scanning [15,16]. Among them, the proportional model by Dempster [4] has been most widely used. These regression equation models are useful for general purpose, but may be limited considering individual characteristics because the parameters have been shown to differ depending on age [7-9,11,14], obesity [3,19], gender [15] and race [16,19]. Moreover, medical imaging methods are limited due to time- and cost-related problems.

Different methods based on the dynamic equation of motion have been introduced to determine individual-customized inertial parameters. Bouisset and Pertuzon [21] developed a mechanical device to measure the moment of inertia of the forearm, and showed the consistency with results obtained from the Dempster's model. However, the measured acceleration included the effects of both passive and active viscoelastic properties that can directly affect the value of the moment of inertia because elbow extensors were active prior to the release. Other studies also showed that the developed method could estimate good results consistent with Dempster's work [22,23]. These methods based on the dynamic equation of motion could easily consider individual characteristics, but might not be widely used as required the self-made devices.

In this study, a simple method based on the dynamic equation of motion was introduced to determine the moment of inertia using a commercial dynamometer, and an optimization technique was utilized to estimate inertial parameters with the determined moment of inertia. To do so, there were two hypotheses that (1) the free-body diagram of the commercial dynamometer can be expressed as a hinge joint model, and (2) any bodies affixed to the dynamometer attachment can be considered as a constant mass segment located at its center of mass. The equation of motion to solve the free-body diagram was established to determine the moment of inertia of the disk-shaped weight with relatively well-defined inertial parameters. The weight was assumed as an ideal solid disk, and the optimization technique was performed to estimate the inertial parameters of the weight. Then, the values of the determined parameters from the developed method were compared with those calculated from theoretical solutions.

\section{Methods}

\subsection{A simple model to determine the moment of inertia}

Most isokinetic dynamometer manufacturers provide attachments, which greatly facilitate an application to various joints. Once the attachment corresponding to the joint to be studied is affixed to the dynamometer shaft, the joint moment can be measured. Therefore, determining the moment of inertia of the attachment has to precede that of a segment. The moment of inertia of a segment can be determined through four simple consecutive steps using a commercial dynamometer, Biodex System 3 Pro (Biodex Medical Systems, USA) in this study. Step (1) corresponds to quasi-static passive movement of the attachment only, Step (2) to fast passive movement of the attachment only, Step (3) to quasistatic passive movement of both the attachment and segment, and Step (4) to fast passive movement of both the attachment and segment. The condition including the attachment only is called as Condition 
A (i.e., Steps (1) and (2)), and the condition including both the attachment and segment is called as Condition B (i.e., Steps (3) and (4)). Free-body diagrams of Conditions A and B can be expressed as a hinge joint model (Figure 1).

If the attachment and segment are considered as a constant mass segment located at its center of mass, their rotation axes would be the same. Then, the equations of motion are determined as Eq. (1):

$$
\begin{aligned}
& \sum M=I \alpha \\
& \text { Condition A: } M_{A}-m_{a} g x_{a} \sin \theta=I_{a} \alpha_{A} \\
& \text { Condition B: } M_{B}-\left(m_{a} g x_{a} \sin \theta+m_{s} g x_{s} \sin \theta\right)=I_{b} \alpha_{B}
\end{aligned}
$$

where $M_{A}$ is the applied moment under Condition A, $m_{a}$ the mass of the attachment, $g$ the gravitational acceleration $\left(=9.81 \mathrm{~m} / \mathrm{s}^{2}\right), x_{a}$ the distance between the axis of the dynamometer shaft (i.e., joint) and the center of mass of the attachment, $\theta$ the angle, $I_{a}$ the moment of inertia of the attachment about the joint and $\alpha_{A}$ the angular acceleration under Condition A. Similarly, $M_{B}$ is the applied moment under Condition $\mathrm{B}, m_{s}$ the mass of the segment, $x_{s}$ the distance between the joint and the center of mass of the segment, $I_{b}$ the sum of each moment of inertia of the attachment and segment about the joint, and $\alpha_{B}$ the angular acceleration under Condition B. If the angular acceleration is not zero, Eq. (1) can be rewritten as:

$$
\begin{aligned}
& \text { Condition A: } I_{a}=\left(M_{A}-m_{a} g x_{a} \sin \theta\right) / \alpha_{A} \\
& \text { Condition B: } I_{b}=\left(M_{B}-\left(m_{a} g x_{a} \sin \theta+m_{s} g x_{s} \sin \theta\right)\right) / \alpha_{B}
\end{aligned}
$$

where $I_{a}$ can be simply determined from Condition A equation if $m_{a} g x_{a} \sin \theta$ is known, since $M_{A}$ and $\alpha_{A}$ are measured. Even though $m_{a}$ and $x_{a}$ are still unknown, $m_{a} g x_{a} \sin \theta$ can be determined from Step (1). Similarly, $I_{b}$ can be also determined from Condition B equation and $\left(m_{a} g x_{a} \sin \theta+\mathrm{m}_{s} g x_{s} \sin \theta\right)$ acquired from Step (3). Once Ia and $\mathrm{Ib}$ are determined through the four simple steps, then the moment of inertia of the segment about the joint $\left(\mathrm{I}_{s}\right)$ can be easily found by Eq. (3).

$$
I_{s}=I_{b}-I_{a}
$$

(a)

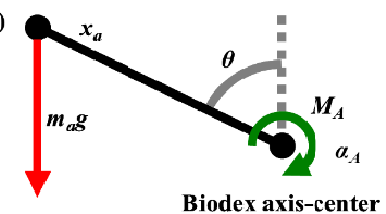

(b)

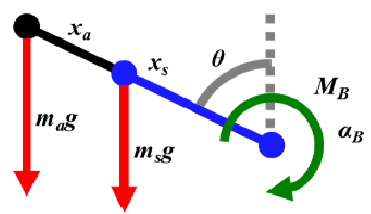

Fig. 1. A free-body diagram of a dynamometer with an attachment only (a) and with both an attachment and a segment (b), where $M$ is the measured moment, $m$ the mass, $g$ the gravitational acceleration, $x$ the distance between the joint and the center of mass, $\theta$ the angle, $I$ the moment of inertia and $\alpha$ the angular acceleration. The subscripts $a, s, A$ and $B$ indicate the attachment, segment, Conditions A and B, respectively. 


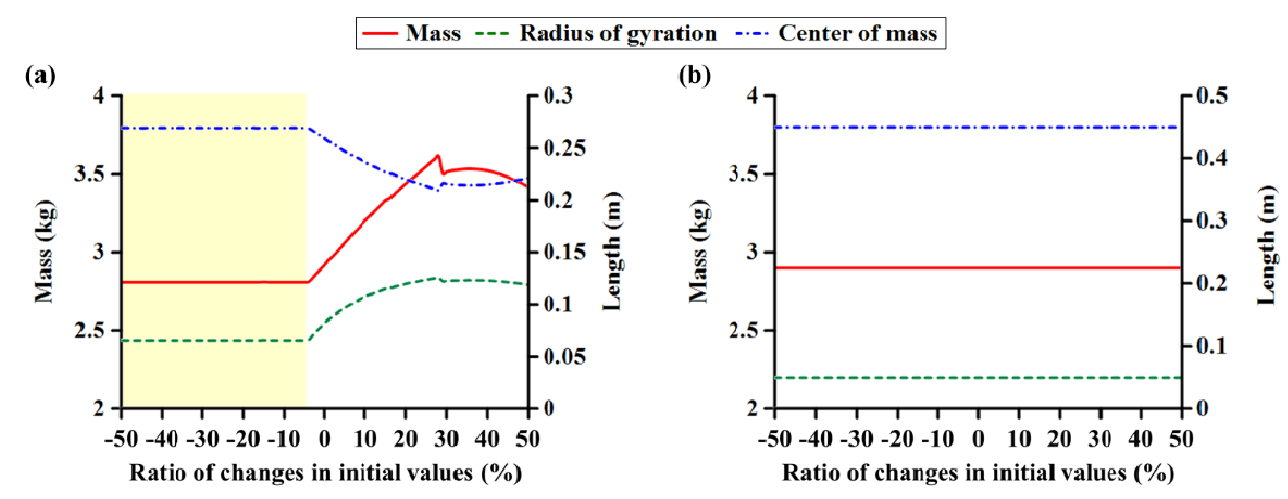

Fig. 2. Optimized inertial parameters given the varied initial values of the elbow attachment (a) and the $3 \mathrm{~kg}$-weight (b). The ivory-shaded box indicates the feasible region in which optimal solution is relatively fixed.

Since the measured values are time-series data, the moment of inertia is defined as the average of the values of the torque divided by the angular acceleration corresponding to each frame.

\subsection{Estimation in other inertial parameters using optimization}

With the determined moment of inertia, the mass, radius of gyration $(\rho)$ and center of mass can be estimated from the optimization technique that minimizes two equations: (1) $I=m \rho^{2}+m x^{2}$ and (2) $M_{g}$ $=m g x$, where $I$ is the determined moment of inertia of either the attachment or segment about the joint, and $M_{g}$ the measured maximum gravitational moment shown as Eq. (4).

$$
\begin{gathered}
\min \left[\left(m x-M_{g} / g\right)^{2}+\left(m \rho^{2}+m x^{2}-I\right)^{2}\right] \\
0.5 m_{0}<m<1.5 m_{0} \\
\text { subject to } 0.5 \rho_{0}<\rho<1.5 \rho_{0} \\
0.5 x_{0}<x<1.5 x_{0}
\end{gathered}
$$

The optimization was performed using the 'fmincon' function in MATLAB (The MathWorks, Inc., USA). Because the 'fmincon' function is designed to find a constrained local minimum of the objective function, it is possible to get different local minimums depending on initial values. Thus, it is important to define feasible regions of reasonable solution since the problem is indeterminate. In order to compensate this, changes in solution were obtained in accordance with varied initial values by $\pm 50 \%$ with the increment of $1 \%$. Mean values within the feasible region in which solutions showed no considerable variations were chosen as final solutions (Figure 2).

\subsection{Evaluation procedures}

To evaluate the feasibility of the developed method, the elbow attachment provided from Biodex System 3 Pro and $3 \mathrm{~kg}$-weight (Figure 3 ) were used. Range of motion (ROM) of the joint was $0^{\circ}$ (away) to $90^{\circ}$ (toward), and then the four steps were performed. The angular speed of $1 \% \mathrm{~s}$ was chosen for quasi-static passive movements. In addition, fast passive movements were applied to make a uni- 
form angular acceleration within the ROM since slow passive movements are not appropriate due to too short acceleration period. Thus, three different passive speeds of 240,270 and $300^{\circ} / \mathrm{s}$ were chosen, because uniform speeds over $240 \%$ s were suitable for our purpose based on our experimental data (Figure 4). Ten trials were performed for each speed, and the data were captured at the sampling rate of $1 \mathrm{kHz}$.

In order to evaluate whether the values of moments of inertia for the elbow attachment $\left(I_{a}\right)$ and for both the attachment and weight $\left(I_{b}\right)$ determined from each speed are not different, one-way ANOVA test was performed with Tukey's HSD post hoc test. All statistical analyses were performed with IBM SPSS Statistics (Version 20, IBM, USA), and the significance level $(p)$ was set to 0.05 . The determined moment of inertia values were compared with those calculated from solutions for ideal models (Table 1). Since the experimentally-determined values correspond to the moment of inertia about the joint, the calculated values about the center of mass were transformed to the values about the joint using the parallel-axis theorem.
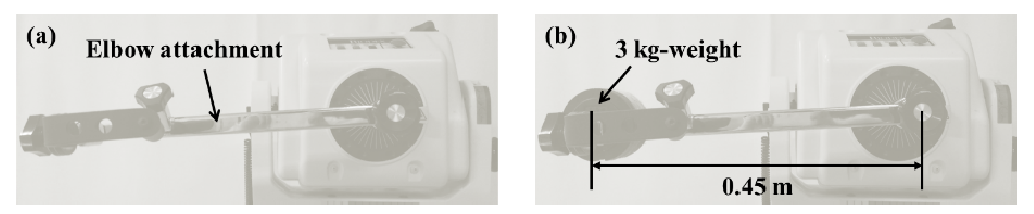

Fig. 3. Experimental setup for the elbow attachment only (a) and both the attachment and 3 kg-weight (b).

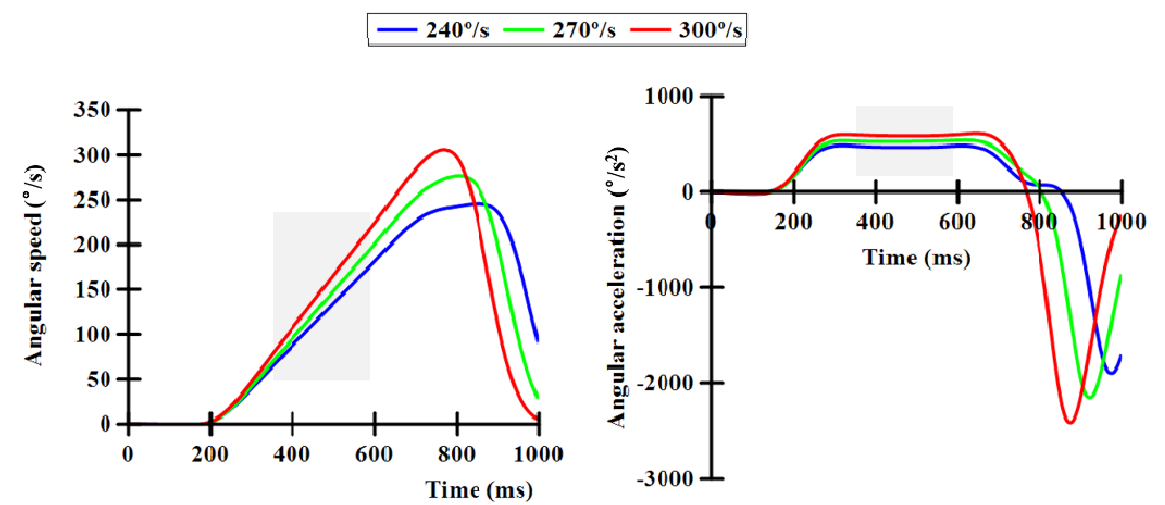

Fig. 4. Three angular speeds and accelerations. Grey-shaded box indicates relatively uniform acceleration period.

Table 1

Information of ideal models

\begin{tabular}{|l|l|l|}
\hline Object & Ideal model & Moment of inertia $\left(\mathrm{kg} \cdot \mathrm{m}^{2}\right)$ \\
\hline $\begin{array}{l}\text { Elbow } \\
\text { attachment }\end{array}$ & $\begin{array}{l}\text { Square tube of } \\
\text { outer edge A, inner edge a, a } \\
\text { length } l, \text { and mass } m\end{array}$ & $\frac{d A^{2} l\left(A^{2}+l^{2}\right)}{12}-\frac{d a^{2} l\left(a^{2}+l^{2}\right)}{12}$ where $d=\frac{A^{2} l-a^{2} l}{m}$ \\
\hline 3 kg-weight & $\begin{array}{l}\text { Solid disk of } \\
\text { radius } r \text { and mass } m\end{array}$ & $\frac{1}{2} m r^{2}$ \\
\hline
\end{tabular}



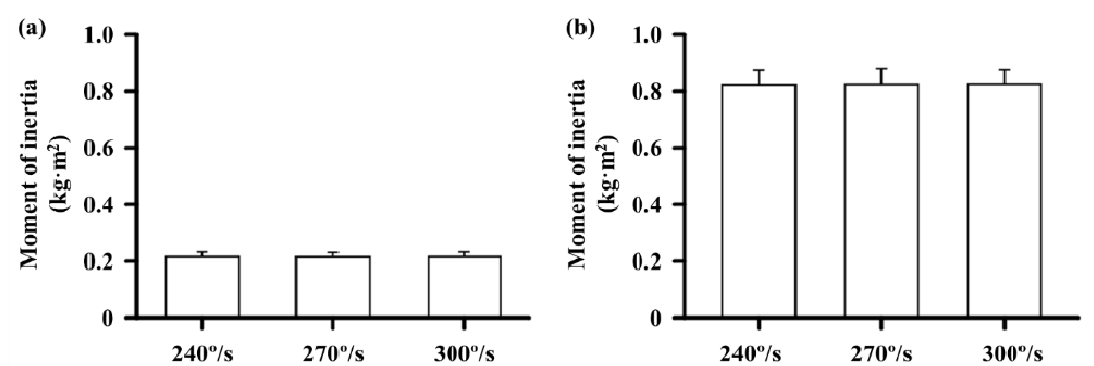

Fig. 5. Moments of inertia of the elbow attachment only (a) and both the attachment and $3 \mathrm{~kg}$-weight (b) at three different speeds. No significant differences were found among the different speeds.

\section{Results}

\subsection{Effects of angular speeds on moments of inertia}

The values of $I_{a}$ were $0.216 \pm 0.017,0.215 \pm 0.016$ and $0.216 \pm 0.017 \mathrm{~kg} \cdot \mathrm{m}^{2}$ at angular speeds of 240,270 and $300 \%$ s, respectively (Figure 5a). This result shows that moments of inertia of the elbow attachment were determined consistently with no significant differences depending on three angular speeds $(p=0.287)$. The values of $I_{b}$ showed $0.821 \pm 0.054,0.823 \pm 0.058$ and $0.824 \pm 0.053 \mathrm{~kg} \cdot \mathrm{m}^{2}$ at angular speeds of 240,270 and $300 \%$ s respectively (Figure $5 \mathrm{~b}$ ), and there were no significant differences $(p=0.276)$. This means that the developed method could be applicable in determining moments of inertia of the weight that was affixed to the elbow attachment.

\subsection{Comparison of optimized inertial parameters with those from ideal models}

The inertial parameters of the elbow attachment were determined as shown in Table 2. The estimated values of the mass and radius of gyration were slightly smaller than those of the theoretical values. However, the center of mass and moment of inertia were overestimated compared with the theoretical values.

The value of the moment of inertia of $3 \mathrm{~kg}$-weight about the joint $\left(I_{w}\right)$ was computed by subtracting the average of all $I_{a}$ values from the average of all $I_{b}$ values, and it was $0.594 \mathrm{~kg} \cdot \mathrm{m}^{2}$. Then, the inertial parameters of the weight were determined as shown in Table 3 . The estimated values were very similar to the theoretical values.

Table 2

Comparison of estimated inertial parameters of the elbow attachment with those calculated from an ideal cuboid model

\begin{tabular}{|c|c|c|c|c|c|c|c|}
\hline & $\begin{array}{l}\text { Outer } \\
\text { edge } \\
(\mathrm{m})\end{array}$ & $\begin{array}{l}\text { Inner } \\
\text { edge } \\
(\mathrm{m})\end{array}$ & $\begin{array}{l}\text { Length } \\
(\mathrm{m})\end{array}$ & $\begin{array}{l}\text { Mass } \\
(\mathrm{kg})\end{array}$ & $\begin{array}{l}\text { Radius of gyration } \\
\text { (m) }\end{array}$ & $\begin{array}{l}\text { Center of mass } \\
\text { (m) }\end{array}$ & $\begin{array}{l}\text { Moment of inertia } \\
\left(\mathrm{kg} \cdot \mathrm{m}^{2}\right)\end{array}$ \\
\hline Ideal & \multirow{2}{*}{$0.03 *$} & \multirow{2}{*}{$0.026^{*}$} & \multirow{2}{*}{$0.45^{*}$} & $2.91 *$ & 0.130 & 0.225 & 0.197 \\
\hline Estimated & & & & 2.81 & 0.065 & 0.269 & 0.216 \\
\hline
\end{tabular}


Table 3

Comparison of estimated inertial parameters of the $3 \mathrm{~kg}$-weight with those calculated from an ideal disk model

\begin{tabular}{|l|l|l|l|l|l|l|}
\hline & $\begin{array}{l}\text { Radius } \\
(\mathrm{m})\end{array}$ & $\begin{array}{l}\text { Length } \\
(\mathrm{m})\end{array}$ & $\begin{array}{l}\text { Mass } \\
(\mathrm{kg})\end{array}$ & $\begin{array}{l}\text { Radius of gyration } \\
(\mathrm{m})\end{array}$ & $\begin{array}{l}\text { Center of mass } \\
(\mathrm{m})\end{array}$ & $\begin{array}{l}\text { Moment of inertia } \\
\left(\mathrm{kg} \cdot \mathrm{m}^{2}\right)\end{array}$ \\
\cline { 1 - 5 } & \multirow{2}{*}{$0.05^{*}$} & \multirow{2}{*}{$0.06^{*}$} & $2.92^{*}$ & 0.035 & 0.45 & 0.595 \\
\cline { 4 - 7 } & & 2.90 & 0.050 & 0.45 & 0.594 \\
\hline
\end{tabular}

\section{Discussion}

In this study, a simple two-step method was introduced to determine the inertial parameters using a commercial dynamometer and an optimization technique. To evaluate the feasibility of the developed method, the inertial parameters of a disk-shaped weight were determined from the method. Then, the determined parameters were compared with those calculated from the theoretical solution. The results showed that the determined moments of inertia from the developed method showed no significant differences depending on three different angular speeds. Moreover, the estimated inertial parameters were comparable with theoretical values. These results seem promising, implying that the developed method could be applicable to determine inertial parameters, such as the mass, center of mass and moment of inertia, of a body segment which is affixed to the attachment.

In the determined inertial parameters of the elbow attachment, the mass was underestimated approximately $0.1 \mathrm{~kg}$ compared to the experimental value. This seems reasonable because the mass corresponding the attachment part near the dynamometer shaft might be negligible. The theoretical position of the center of mass is located at $0.225 \mathrm{~m}$ far from the joint, but the estimated one at $0.269 \mathrm{~m}$. Moreover, the measured moment of inertia was greater than the ideal one. These may be acceptable, because the real elbow attachment has not the same shape as an ideal square cube; indeed, there are grabbling parts at the furthermost end of the attachment. The estimated inertial parameters of the $3 \mathrm{~kg}$-weight were very similar to the theoretical ones, showing the small difference of $0.001 \mathrm{~kg} \cdot \mathrm{m}^{2}$ only. This implies that the selection of disk-like weight might be appropriate to evaluate the developed method, and also supports that the developed method has the potential to determine segmental inertial parameters of an individual object.

There are some limitations in this study. First, whether the developed method is still valid to the living subject was not confirmed. However, this preliminary study should be conducted prior to the confirmation whether the developed method is valid to rigid bodies. Based on our results, the moment of inertia of the elbow attachment or both the attachment and $3 \mathrm{~kg}$-weight was determined with no significant differences irrespective of angular speeds. From this, it is expect that the developed method could be valid to rigid bodies at least. Further studies would be required to confirm whether the developed method is applicable to the human body segment. Human body segments consist of various tissues, especially muscles, which are deformable depending on their activations. In this regard, some researchers suggested that it is needed to avoid active viscoelastic effects of muscles on joint stiffness $[22,24]$. Indeed, since human being can regulate joint coordination by the muscular stiffness [25], it is reasonable that measured data can include intrinsic muscle properties and extrinsic muscle effects, i.e. muscle activities, as well. In further studies with living subjects, thus, it seems that there is a need for a muscle activity monitoring using an electromyography or a fixing of the segment and attachment using a bandage to assist the exclusion of various human factors.

In this study, only the elbow attachment was used. If we are interested in the lower leg, we can easily determine the moment of inertia of the lower leg through the same procedure only by replacing the 
elbow attachment with the knee attachment. Instead, our method may be limited to determine the moment of inertia of proximal segments in terms of safety (i.e., too fast angular speed for torso joints). Fortunately, forward dynamics studies in which inertial parameters are considered to be more important have been focused on distal segments such as elbow [26] and knee [27] joints rather than proximal ones. Hence, it is expected that the developed method have the potential to estimate inertial parameters of a human body segment and to improve the accuracy and reliability of human dynamics studies.

\section{Acknowledgment}

This research was financially supported by the Ministry of Education (MOE) and National Research Foundation of Korea (NRF) through the Human Resource Training Project for Regional Innovation (No. 2013H1B8A2032194), and a grant from the Korean Health Technology R \& D Project, Ministry of Health \& Welfare, Republic of Korea (HI10C2017 (A102062)).

\section{References}

[1] D.J. Pearsall and P.A. Costigan, The effect of segment parameter error on gait analysis results, Gait Posture 9 (1999), 173-183.

[2] J.G. Andrews and S.P. Mish, Methods for investigating the sensitivity of joint resultants to body segment parameter variations, J. Biomech. 29 (1996), 651-654.

[3] S. Chen, H. Hsieh, T. Lu and C. Tseng, A method for estimating subject-specific body segment inertial parameters in human movement analysis, Gait Posture 33 (2011), 695-700.

[4] W.T. Dempster, Space requirements of the seated operator, in: Space requirements of the seated operator, WrightPatterson Air Force Base, Ohio, 1955, pp. 55-159.

[5] M.R. Forwood, R.J. Neal and B.D. Wilson, Scaling segmental moments of inertia for individual subjects, J. Biomech. 18 (1985), 755-761.

[6] P. De Leva, Adjustments to Zatsiorsky-Seluyanov's segment inertia parameters, J. Biomech. 29 (1996), $1223-1230$.

[7] A. Matsuo, H. Ozawa, K. Goda and T. Fukunaga, Moment of inertia of whole body using an oscillating table in adolescent boys, J. Biomech. 28 (1995), 219-223.

[8] J. Muri, S.L. Winter and J.H. Challis, Changes in segmental inertial properties with age, J. Biomech. 41 (2008), 18091812.

[9] R.K. Jensen, Changes in segment inertia proportions between 4 and 20 years, J. Biomech. 22 (1989), 529-536.

[10] M.R. Yeadon and M. Morlock, The appropriate use of regression equations for the estimation of segmental inertia parameters, J. Biomech. 22 (1989), 683-689.

[11] M.K. Lebiedowska and A. Polisiakiewicz, Changes in the lower leg moment of inertia due to child's growth, J. Biomech. 30 (1997), 723-728.

[12] R.N. Hinrichs, Regression equations to predict segmental moments of inertia from anthropometric measurements: An extension of the data of chandler et al. (1975), J. Biomech. 18 (1985), 621-624.

[13] T.R. Ackland, B.A. Blanksby and J. Bloomfield, Inertial characteristics of adolescent male body segments, J. Biomech. 21 (1988), 319-327.

[14] K. Schneider and R.F. Zernicke, Mass, center of mass, and moment of inertia estimates for infant limb segments, J. Biomech. 25 (1992), 145-148.

[15] Y. Ma, K. Lee, L. Li and J. Kwon, Nonlinear regression equations for segmental mass-inertial characteristics of Korean adults estimated using three-dimensional range scan data, Appl. Ergon. 42 (2011), 297-308.

[16] Y. Ma, J. Kwon, Z. Mao, K. Lee, L. Li and H. Chung, Segment inertial parameters of Korean adults estimated from three-dimensional body laser scan data, Int. J. Ind. Ergonom. 41 (2011), 19-29.

[17] T.R. Ackland, P.W. Henson and D.A. Bailey, The uniform density assumption: Its effect upon the estimation of body segment inertial parameters, J. Appl. Biomech. 4 (1988), 146-155. 
[18] P.E. Martin, M. Mungiole, M.W. Marzke and J.M. Longhill, The use of magnetic resonance imaging for measuring segment inertial properties, J. Biomech. 22 (1989), 367-369.

[19] T.C. Pataky, V.M. Zatsiorsky and J.H. Challis, A simple method to determine body segment masses in vivo: Reliability, accuracy and sensitivity analysis, Clin. Biomech. 18 (2003), 364-368.

[20] M. Mungiole and P.E. Martin, Estimating segment inertial properties: Comparison of magnetic resonance imaging with existing methods, J. Biomech. 23 (1990), 1039-1046.

[21] S. Bouisset and I. Pertuzon, Experimental determination of the moment of inertia of limb segments, in: International Seminar on Biomechanics 1st, Karger Basel, Zurich, Switzerland, 1968, pp. 106-109.

[22] J.H. Allum and L.R. Young, The relaxed oscillation technique for the determination of the moment of inertia of limb segments, J. Biomech. 9 (1976), 21-25.

[23] A.J. Peyton, Determination of the moment of inertia of limb segments by a simple method, J. Biomech. 19 (1986), 405410.

[24] H. Hatze, A new method for the simultaneous measurement of the moment of inertia, the damping coefficient and the location of the centre of mass of a body segment in situ, Euro. J. Appl. Physio. 34 (1975), 217-226.

[25] T.R. Nichols, The contributions of muscles and reflexes to the regulation of joint and limb mechanics, Clin. Orthop. Relat. R. 403S (2002), S43-S50.

[26] L. Li, K.Y. Tong, X.L. Hua, L.K. Hung and T.K.K. Koo, Incorporating ultrasound-measured musculotendon parameters to subject-specific EMG-driven model to simulate voluntary elbow flexion for persons after stroke, Clin. Biomech. 24 (2009), 101-109.

[27] D.G. Thelen, E.S. Chumanov, M.A. Sherry and B.C. Heiderscheit, Neuromusculoskeletal models provide insights into the mechanisms and rehabilitation of hamstring strains, Exerc. Sport Sci. Rev. 34 (2006), 135-141. 